\title{
Some universal limits for nonhomogeneous birth and death processes
}

\author{
A. Zeifman $\cdot$ S. Leorato $\cdot$ E. Orsingher $\cdot$ Ya. Satin · \\ G. Shilova
}

Received: 26 May 2004 / Accepted: 16 August 2005

(C) Springer Science + Business Media, Inc. 2006

\begin{abstract}
In this paper we consider nonhomogeneous birth and death processes (BDP) with periodic rates. Two important parameters are studied, which are helpful to describe a nonhomogeneous BDP $X=X(t), t \geq 0$ : the limiting mean value (namely, the mean length of the queue at a given time $t$ ) and the double mean (i.e. the mean length of the queue for the whole duration of the BDP). We find conditions of existence of the means and determine bounds for their values, involving also the truncated BDP $X_{N}$. Finally we present some examples where these bounds are used in order to approximate the double mean.
\end{abstract}

Keywords Birth and death rates - Kolmogorov differential equations $\cdot$ Logarithmic norm $\cdot$ Exponential stability

AMS Subject Classification: Primary: 60J27; Secondary: $60 \mathrm{~K} 25,34 \mathrm{~A} 30$

\section{Introduction}

Homogeneous and nonhomogeneous birth and death processes have a great importance for many fields of ap-

\author{
A. Zeifman $(\bowtie)$ \\ Vologda State Pedagogical University, S. Orlova, 6, Vologda, \\ Russia; Vologda Science Coordination Centre CEMI RAS. \\ Vologda, Russia \\ e-mail: zai@uni-vologda.ac.ru \\ Ya. Satin · G. Shilova \\ Vologda State Pedagogical University, S. Orlova, 6, Vologda, \\ Russia \\ S. Leorato $\cdot$ E. Orsingher \\ University of Rome 'La Sapienza', P.le A. Moro, 5, Rome, Italy
}

plied probability. Nonhomogeneous versions of the birth and death process have an important role in queueing theory.

It is extremely difficult to obtain general results for arbitrary forms of the birth and death rates and therefore we must content ourselves in obtaining various types of approximations.

In the literature there exist some papers devoted to the problem of approximating BDP (see [2] for diffusions approximations and [6] and [8]). Estimates concerning the distance between truncated and infinite models for homogeneous BDP are presented in [9]. Some queueing applications of periodic BDP are given in [7].

In the case of rates with periodic behavior we study here two parameters, that is the limiting mean value and the "double mean".

We can interpret the first parameter as the mean value, at arbitrary time $t$, of the number of customers queueing up before some device (e.g. bancomat counter).

The second characteristic represents the mean number of customers in the queue for the whole duration of the service.

Our approach is based on the method introduced by Gnedenko and Makarov (see [3]) and successively worked out by one of the authors in [10] and [13].

The method based on the application of the logarithmic norm of operators and the corresponding estimates is dealt with in Daletsky and Krein (see [1]). An important aspect of the analysis is the choice of the suitable transformation of the reduced matrix of the process rates.

This procedure in the simplest cases is accurately examined in [4] and its description in the general case of nonhomogeneous BDP and the related applications to the study of queueing systems is presented in [5]. 


\section{Basic results}

Let $X=X(t), t \geq 0$ be a nonhomogeneous birth and death process (BDP) on the state space $E=(0,1,2, \ldots)$ and with birth rates $\lambda_{n}=\lambda_{n}(t), t \geq 0$ and death rates $\mu_{n}=\mu_{n}(t), n \in$ $E$. This means that

$$
\begin{aligned}
& P\{X(t+h)=j \mid X(t)=i\} \\
& \quad= \begin{cases}\lambda_{i}(t) \cdot h+o(h, t, i), & \text { if } j=i+1 \\
\mu_{i}(t) \cdot h+o(h, t, i), & \text { if } j=i-1 \\
1-\left(\lambda_{i}(t)+\mu_{i}(t)\right) \cdot h+o(h), & \text { if } j=i \\
o(h, t, i), & \text { if }|i-j|>1\end{cases}
\end{aligned}
$$

for $h>0$ and with $\lim _{h \downarrow 0} \frac{o(h, t, i)}{h}=0$ uniformly with respect to $i \in E$. This condition plays an essential role in deriving the Kolmogorov differential system in the space $l_{1}$.

The nonstationarity of $X$ depends on the fact that the rates $\mu_{i}(t)$ and $\lambda_{i}(t)$ are functions of time and depend on the current size of the queue.

Let

$$
\begin{array}{r}
p_{i j}(s, t)=\operatorname{Pr}\{X(t)=j \mid X(s)=i\} \\
\text { for } i, j \in E, 0 \leq s \leq t
\end{array}
$$

be the transition probability function of the process $X=X(t)$ and

$p_{i}(t)=\operatorname{Pr}\{X(t)=i\} \quad$ for $i \in E, t>0$

be the state probabilities.

We denote by $\mathbf{p}(t)=\left(p_{0}(t), p_{1}(t), p_{2}(t), \ldots\right)^{T}, t>0$ the column vector of state probabilities and by $\mathbf{A}(t)=$ $\left\{a_{i j}(t), t \geq 0\right\}$ the matrix related to (2.1) where

$a_{i j}(t)= \begin{cases}\lambda_{i-1}(t), & \text { if } j=i-1 \\ \mu_{i+1}(t), & \text { if } j=i+1 \\ -\left(\lambda_{i}(t)+\mu_{i}(t)\right), & \text { if } j=i \\ 0, & \text { if otherwise. }\end{cases}$

The probabilistic dynamics of the process is represented by the forward Kolmogorov differential system:

$$
\frac{d \mathbf{p}}{d t}=\mathbf{A}(t) \mathbf{p}, \quad \mathbf{p}=\mathbf{p}(t), \quad t \geq 0 .
$$

The Cauchy problem formed by (2.3) with the initial conditions $\mathbf{p}(s)$ has the following solution

$$
\mathbf{U}(t, s)=\left\{p_{i j}(t, s)\right\}_{i, j=0}^{\infty} \quad \text { for } \quad t>s,
$$

where $p_{i, j}(t, s)=\operatorname{Pr}\{X(t)=j \mid X(s)=i$. $\}$.

Throughout the whole paper we use the $l_{1}$-norm for vectors $\mathbf{x}$ that is $\|x\|_{1}=\sum_{i \in E}\left|x_{i}\right|$ and

$\|\mathbf{B}\|=\sup _{j \in E} \sum_{i \in E}\left|b_{i j}\right|=\sup _{j \in E}\left\|\mathbf{b}_{j}\right\|_{1}$

where $\mathbf{x}=\left(x_{0}, x_{1}, \ldots\right)^{T}$ and $\mathbf{B}=\left\{b_{i j}\right\}_{i, j=0}^{\infty}$.

Let $\Omega=\left\{\mathbf{x}: \mathbf{x} \geq 0,\|\mathbf{x}\|_{1}=1\right\}$. We shall restrict ourselves to birth and death processes whose rates have the following form:

$\lambda_{n}(t)=v_{n} a(t), \quad \mu_{n}(t)=\eta_{n} b(t), \quad t \geq 0, \quad n \in E$,

with the assumptions that the rates are bounded, i.e.

$0<\eta_{n} \leq M, n>0$ and $0 \leq v_{n} \leq L, n \geq 0$

and, obviously, $\eta_{0}=0$.

We clearly assume that the functions $a=a(t)$ and $b=$ $b(t)$ are non-negative, 1-periodic and bounded, with bounds

$a(t) \leq a, \quad b(t) \leq b$.

Conditions (2.4)-(2.6) guarantee (see [1]) the boundedness and integrability of the operator function $A(t)$ in the space of sequences $\ell_{1}$, where

$\|A(t)\|_{1}=2 \sup _{i}\left(\lambda_{i}(t)+\mu_{i}(t)\right) \leq 2(L a+M b)$.

We remark that conditions (2.4)-(2.6) are not used in the proofs of the results below. These conditions are introduced with the purpose of guaranteeing the integrability of the operator function $A(t)$ and can be weakened. For example it is possible to replace (2.4) either with the condition that $A(t)$ is itself integrable or with the alternative condition that the rates are linear combinations of a finite number of integrable functions.

In [1] it is shown that the Cauchy problem for linear differential equations in Banach spaces with bounded and integrable operator functions has unique solutions for arbitrary initial conditions. This means that, under our assumptions, the existence and uniqueness of the solution do not pose any problem.

We shall study the following mean values

$E_{\mathbf{p}(0)}\{X(t)\}=\sum_{k \in E} E\{X(t) \mid X(0)=k\} p_{k}(0)$,

and the conditional mean value

$E\{X(t) \mid X(0)=k\}$. 
For our further analysis we need the following quantities. Let $1=d_{-1}=d_{0} \leq d_{1} \leq \cdots$ and define

$$
\begin{aligned}
\alpha_{k}(t)= & \lambda_{k}(t)+\mu_{k+1}(t)-\frac{d_{k+1}}{d_{k}} \lambda_{k+1}(t) \\
& -\frac{d_{k-1}}{d_{k}} \mu_{k}(t), k \geq 0,
\end{aligned}
$$

and consequently

$$
\begin{aligned}
\alpha(t) & =\inf _{k \geq 0} \alpha_{k}(t), \quad \alpha^{*}=\int_{0}^{1} \alpha(t) d t, \\
M_{0} & =\sup _{|t-s| \leq 1} \int_{s}^{t} \alpha(u) d u, \quad M=e^{M_{0}+\alpha^{*}}, \\
W & =\inf _{k} \frac{\sum_{i=0}^{k-1} d_{i}}{k}
\end{aligned}
$$

We remark that our aim here is the evaluation of estimates for the speed of convergence of the means. Therefore the conditions appearing in the next Theorem are not necessary but sufficient for the existence of the mean of the limiting regime.

The results of next theorems are formulated in terms of the auxiliary sequences $d_{i}, i \geq 1$, which do not possess any probabilistic meaning. A detailed analysis of their properties is given in [4]. We note that they are a sort of counterpart of the Lyapunov functions.

Theorem 1. Let a birth and death process with rates $\lambda_{k}(t)$ and $\mu_{k}(t), k \geq 0$ be given. Let us assume that there exists a sequence $\left\{d_{j}\right\}$ such that the number $\alpha^{*}$ defined above is strictly positive.

We also assume that the numbers $d_{j}$ grow sufficiently fast so that $\inf _{k \geq 1} \frac{d_{k-1}}{k}=\omega>0$.

Under all these conditions there exists the limit

$\lim _{t \rightarrow \infty}|E\{X(t) \mid X(0)=k\}-\phi(t)|=0$

for all $k$ and for some 1-periodic function $\phi(t)$.

We claim also that, for $k=0$, the following upper bound holds

$|E\{X(t) \mid X(0)=k\}-\phi(t)| \leq \frac{a \nu_{0} M^{2}}{W \alpha^{*}} e^{-\alpha^{*} t}$.
Proof. Equation (2.3) in explicit form reads

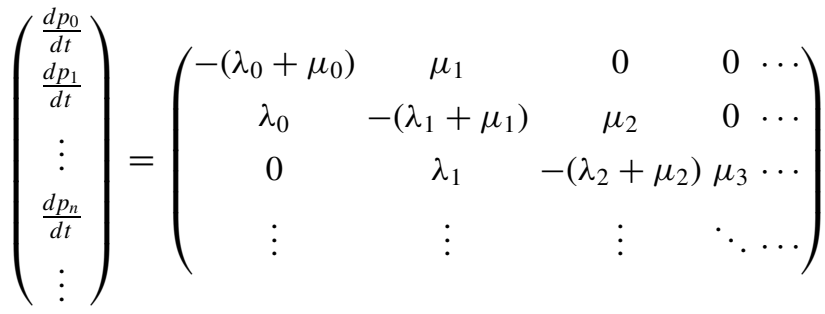

$$
\times\left(\begin{array}{c}
p_{0} \\
p_{1} \\
\vdots \\
p_{n} \\
\vdots
\end{array}\right)
$$

By introducing (see [10-13]), $p_{0}(t)=1-\sum_{i=1}^{\infty} p_{i}(t)$, the second scalar equation

$\frac{d p_{1}}{d t}=\lambda_{0} p_{0}-\left(\lambda_{1}+\mu_{1}\right) p_{1}+\mu_{2} p_{2}$,

becomes

$\frac{d p_{1}}{d t}=\lambda_{0}-\lambda_{0} \sum_{i=1}^{\infty} p_{i}-\left(\lambda_{1}+\mu_{1}\right) p_{1}+\mu_{2} p_{2}$

so that the system (2.12) writes

$\left(\begin{array}{c}\frac{d p_{1}}{d t} \\ \frac{d p_{2}}{d t} \\ \vdots \\ \frac{d p_{n}}{d t} \\ \vdots\end{array}\right)$

$=\left(\begin{array}{cccccc}-\left(\lambda_{0}+\lambda_{1}+\mu_{1}\right) & \mu_{2}-\lambda_{0} & -\lambda_{0} & -\lambda_{0} & \cdots & \cdots \\ \lambda_{1} & -\left(\lambda_{2}+\mu_{2}\right) & \mu_{3} & 0 & 0 & \cdots \\ 0 & \lambda_{2} & -\left(\lambda_{3}+\mu_{3}\right) & \mu_{4} & 0 & \cdots \\ \vdots & \vdots & \vdots & \vdots & \vdots & \ddots\end{array}\right)$

$\times\left(\begin{array}{c}p_{1} \\ p_{2} \\ \vdots \\ p_{n} \\ \vdots\end{array}\right)+\left(\begin{array}{c}\lambda_{0} \\ 0 \\ \vdots \\ 0 \\ \vdots\end{array}\right)$ 
or otherwise

$\frac{d \mathbf{z}(t)}{d t}=B(t) \mathbf{z}(t)+\mathbf{f}(t)$

This is a linear nonhomogeneous differential system the solution of which can be written as

$\mathbf{z}(t)=V(t, 0) \mathbf{z}(0)+\int_{0}^{t} V(t, z) \mathbf{f}(z) d z$

where $V(t, z)$ is the Cauchy operator of (2.14). The corresponding theory is treated in detail in the book by Daletsky and Krein. We furthermore remark that, if $B$ does not depend on $t$, then the Cauchy operator possesses the explicit simple form $V(t, s)=e^{(t-s) B}$.

There is the following simple relationship between pairs, $\mathbf{z}^{(i)}=\mathbf{z}^{(i)}(t), t \geq 0, i=1,2$, of solutions of (2.14) and pairs of solutions of (2.3), $\mathbf{p}^{(i)}=\mathbf{p}^{(i)}(t), t \geq 0, i=1,2$ :

$$
\begin{aligned}
& \left\|\mathbf{p}^{(1)}-\mathbf{p}^{(2)}\right\|_{1} \\
& =\left|p_{0}^{(1)}-p_{0}^{(2)}\right|+\sum_{i \geq 1}\left|p_{i}^{(1)}-p_{i}^{(2)}\right| \\
& =\left|1-\sum_{i \geq 1} p_{i}^{(1)}-\left(1-\sum_{i \geq 1} p_{i}^{(2)}\right)\right| \\
& \quad+\left\|\mathbf{z}^{(1)}-\mathbf{z}^{(2)}\right\|_{1}=\left|\sum_{i \geq 1}\left(p_{i}^{(2)}-p_{i}^{(1)}\right)\right| \\
& \quad+\left\|\mathbf{z}^{(1)}-\mathbf{z}^{(2)}\right\|_{1} \leq \sum_{i \geq 1}\left|p_{i}^{(2)}-p_{i}^{(1)}\right| \\
& \quad+\left\|\mathbf{z}^{(1)}-\mathbf{z}^{(2)}\right\|_{1}=2\left\|\mathbf{z}^{(1)}-\mathbf{z}^{(2)}\right\|_{1}, \quad t \geq 0 .
\end{aligned}
$$

Consequently,

$$
\begin{array}{r}
\left\|\mathbf{z}^{(1)}-\mathbf{z}^{(2)}\right\|_{1} \leq\left\|\mathbf{p}^{(1)}-\mathbf{p}^{(2)}\right\|_{1} \leq 2\left\|\mathbf{z}^{(1)}-\mathbf{z}^{(2)}\right\|_{1}, \\
t \geq 0,
\end{array}
$$

which will be used in the study of stability and ergodicity.

Consider the matrix

$D=\left(\begin{array}{cccc}d_{0} & d_{0} & d_{0} & \ldots \\ 0 & d_{1} & d_{1} & \ldots \\ 0 & 0 & d_{2} & \ldots \\ \vdots & \vdots & \ddots & \ddots\end{array}\right)$

and the space of sequences

$\ell_{1 D}=\left\{\mathbf{z}^{T}=\left(p_{1}, p_{2}, \ldots\right):\|\mathbf{z}\|_{1 D}=\|D \mathbf{z}\|_{1}<\infty\right\}$,

as in [13].
We have

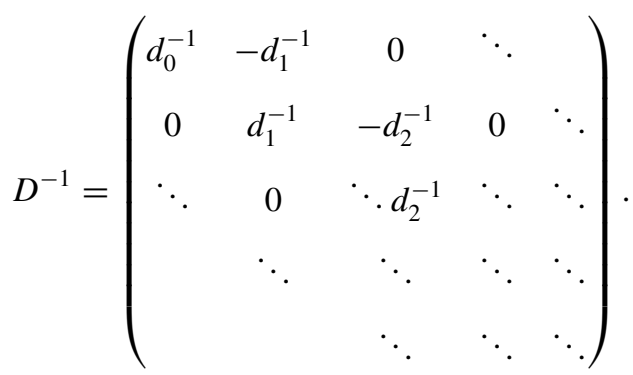

By applying this transformation to the matrix $B(t)$ in (2.14), we arrive at the matrix $D B(t) D^{-1}$

$$
\begin{aligned}
& D B(t) D^{-1} \\
& =\left(\begin{array}{ccccc}
-\left(\lambda_{0}+\mu_{1}\right) & d_{0} \cdot d_{1}^{-1} \cdot \mu_{1} & 0 & & \cdots \\
d_{1} \cdot d_{0}^{-1} \cdot \lambda_{1} & -\left(\lambda_{1}+\mu_{2}\right) & d_{1} \cdot d_{2}^{-1} \cdot \mu_{2} & 0 & \\
0 & d_{2} \cdot d_{1}^{-1} \cdot \lambda_{2} & \ddots & \ddots & \ddots \\
\vdots & 0 & \ddots & \ddots & \ddots \\
& \cdots & \ddots & \ddots & \ddots
\end{array}\right)
\end{aligned}
$$

We recall the definition of the logarithmic norm that was proposed for finite-dimensional spaces by Lozinskij and generalized to Banach spaces by Daletsky and Krein, see for instance [13].

Definition 1. Let $B(t), t \geq 0$ be a one-parameter family of bounded linear operators on a Banach space $\mathcal{B}$ and let $I$ denote the identity operator. For each $t \geq 0$, the number

$\gamma(B(t))=\lim _{h \rightarrow+0} \frac{\|I+h B(t)\|-1}{h}$

is called the logarithmic norm of the operator $B(t)$.

The logarithmic norm of the matrix $B(t)=\left\{b_{i j}(t)\right\}, t \geq 0$ corresponding to a linear operator on the vector space $\mathcal{B}$ equipped with $\ell_{1}$-norm, is

$\gamma(B(t))=\sup _{j}\left(b_{j j}(t)+\sum_{i \neq j}\left|b_{i j}(t)\right|\right), \quad t \geq 0$.

Let $B(t)$ be an operator function corresponding to Eq. (2.13). Then the logarithmic norm of the operator $B(t)$ is related to 
the Cauchy operator $V(t, s), 0 \leq s \leq t$ of the system

$\frac{d \mathbf{x}}{d t}=B(t) \mathbf{x}, \quad t \geq 0$

by

$\gamma(B(t))=\lim _{h \rightarrow+0} \frac{\|V(t+h, t)\|-1}{h}, \quad t \geq 0$.

From the latter one can deduce the following bounds on the $\mathcal{B}$-norm of the Cauchy operator $V(t, s), 0 \leq s \leq t$ :

$e^{-\int_{s}^{t} \gamma(-B(\tau)) d \tau} \leq\|V(t, s)\| \leq e^{\int_{s}^{t} \gamma(B(\tau)) d \tau}, \quad 0 \leq s \leq t$.

Moreover, for any solution $\mathbf{x}(t) \in \mathcal{B}, t \geq 0$ of (2.22) we have

$\|\mathbf{x}(t)\| \geq e^{-\int_{s}^{t} \gamma(-B(\tau)) d \tau}\|\mathbf{x}(s)\|$.

We will also make use of the fact that if $\mathcal{B}$ is a vector space with norm $\ell_{1}$ and all diagonal elements of $B$ are non-negative then, by (2.21)

$\gamma(B(t))=\sup _{j} \sum_{i} b_{i j}(t), \quad t \geq 0$

and, a fortiori, for any solution $\mathbf{x}(t), t \geq 0$ of (2.22), s.t. $\mathbf{x}(s) \geq \mathbf{0}$, we have

$\|\mathbf{x}(t)\| \geq e^{\int_{s}^{t} \inf _{j} \sum_{i} b_{i j}(\tau) d \tau}\|\mathbf{x}(s)\|, \quad 0 \leq s \leq t$.

If $\mathcal{B}$ is endowed with norm $\ell_{1 D}$, from (2.24) we have that

$\|V(t, s)\|_{1 D} \leq \exp \left\{\int_{s}^{t} \gamma(B(\tau))_{\ell_{1 D}} d \tau\right\}$

and, by (2.26)

$\gamma(B(t))_{\ell_{1 D}}=\gamma\left(D B(t) D^{-1}\right)=-\inf _{k} \alpha_{k}(t)=-\alpha(t)$.

In view of (2.26) and having in mind (2.28) we obtain the following inequality

$$
\begin{aligned}
& \left\|\mathbf{p}^{(1)}(t)-\mathbf{p}^{(2)}(t)\right\|_{1 D} \\
& \quad \leq e^{-\int_{s}^{t} \alpha(u) d u}\left\|\mathbf{p}^{(1)}(s)-\mathbf{p}^{(2)}(s)\right\|_{1 D}, \quad 0 \leq s \leq t,
\end{aligned}
$$

for all $\mathbf{p}^{(i)}(s) \in \ell_{1 D}, \quad i=1,2$. Now, periodicity of $\alpha(t)$ and estimate (2.29) imply the exponential stability of Eq. (2.14) and the existence of a 1-periodic solution of this equation $\mathbf{q}(t) \in \ell_{1 D}$. Hence, the respective $\pi(t)=\left(\begin{array}{c}1-\sum_{i \geq 1} q_{i}(t) \\ \mathbf{q}(t)\end{array}\right) \in \ell_{1 D}$ represents the limiting 1-periodic regime of $X(t)$, where $X(t)$ is the BDP considered.

Let $\ell_{1 E}$ be the space of sequences

$$
\ell_{1 E}=\left\{\mathbf{z}=\left(p_{1}, p_{2}, \ldots\right)^{T}:\|\mathbf{z}\|_{1 E}=\sum n\left|p_{n}\right|<\infty\right\}
$$

We have for any vector $\mathbf{z}=\left(p_{1}, p_{2}, \ldots\right)^{T} \in l_{1 D}$ the following bound:

$$
\begin{aligned}
\|\mathbf{z}\|_{1 E} & =\sum_{n \geq 1} n\left|p_{n}\right|=\sum_{n \geq 1} \frac{n}{d_{n-1}} d_{n-1}\left|p_{n}\right| \leq \omega^{-1} \sum_{n \geq 1} d_{n-1}\left|p_{n}\right| \\
& =\omega^{-1} \sum_{n \geq 1} d_{n-1}\left|\sum_{i \geq n} p_{i}-\sum_{i \geq n+1} p_{i}\right| \\
& \leq \omega^{-1} \sum_{n \geq 1} d_{n-1}\left(\left|\sum_{i \geq n} p_{i}\right|+\left|\sum_{i \geq n+1} p_{i}\right|\right) \\
& \leq 2 \omega^{-1} \sum_{n \geq 0} d_{n}\left|\sum_{i \geq n} p_{i}\right| \leq 2 \omega^{-1}\|\mathbf{z}\|_{1 D}
\end{aligned}
$$

Therefore, as $\mathbf{q}(t) \in \ell_{1 D}$, there exists the limit 1-periodic mean $\phi(t)=\sum n \pi_{n}(t)<\infty$.

Let $\mathbf{p}(0)=\left(p_{0}(0), p_{1}(0), \ldots, p_{n}(0), \ldots\right)^{T}=\mathbf{e}_{0}=(1,0$, $0, \ldots)^{T}$, and put $\mathbf{z}(0)=\left(\pi_{1}(0)-p_{1}(0), \ldots\right)^{T}$. Then $\mathbf{z}(0) \geq$ $\mathbf{0}$, and if $\mathbf{v}(t)=D \mathbf{z}(t)$, then one has $\mathbf{v}(0) \geq \mathbf{0}$. We have that $\mathbf{v}(t)$ satisfies the homogeneous differential system

$\frac{d \mathbf{v}}{d t}=D B(t) D^{-1} \mathbf{v}, \quad t \geq 0$.

All non-diagonal elements of the matrix $D B(t) D^{-1}$ are nonnegative for all $t \geq 0$ according to (2.19). Then $\mathbf{v}(t) \geq \mathbf{0}$ for all $t \geq 0$.

Therefore, $\sum_{i \geq k} z_{i}(t) \geq 0$ for any $t \geq 0$ and any $k$. Now, the following estimate holds:

$$
\begin{aligned}
\|\mathbf{z}\|_{1 D} & =\sum_{k=0}^{\infty} d_{k} \sum_{i \geq k+1} p_{i}=\sum_{i=1}^{\infty} i p_{i} \frac{\sum_{k=0}^{i-1} d_{k}}{i} \\
& \geq \inf _{i \geq 1} \frac{\sum_{k=0}^{i-1} d_{k}}{i} \sum_{h=1}^{\infty} h p_{h}=\mathrm{W}\|\mathbf{z}\|_{1 E} .
\end{aligned}
$$

Then, by (2.29) and (2.33) we obtain

$$
\begin{aligned}
& |E\{X(t) \mid X(0)=0\}-\phi(t)| \\
& \quad \leq\|\mathbf{z}(t)\|_{1 E} \leq \mathbf{W}^{-1}\|\boldsymbol{\pi}(t)-\mathbf{p}(t)\|_{1 D}=\mathbf{W}^{-1}\|\mathbf{z}(t)\|_{1 D}
\end{aligned}
$$




$$
\begin{aligned}
& \leq \mathbf{W}^{-1} \exp \left(-\int_{0}^{t} \alpha(s) d s\right)\|\mathbf{z}(0)\|_{1 D} \\
& =\mathbf{W}^{-1} \exp \left(-\int_{0}^{t} \alpha(s) d s\right)\|\boldsymbol{\pi}(0)-\mathbf{p}(0)\|_{1 D} .
\end{aligned}
$$

By writing

$$
\begin{aligned}
\int_{0}^{t} \alpha(s) d s= & \int_{0}^{\lfloor t\rfloor} \alpha(s) d s+\int_{\lfloor t\rfloor}^{t} \alpha(s) d s \quad \text { (by periodicity) } \\
= & \lfloor t\rfloor \int_{0}^{1} \alpha(s) d s+\int_{\lfloor t\rfloor}^{t} \alpha(s) d s \\
= & \lfloor t\rfloor \alpha^{*}+\int_{\lfloor t\rfloor}^{t} \alpha(s) d s=\alpha^{*}(t-t+\lfloor t\rfloor) \\
& +\int_{\lfloor t\rfloor}^{t} \alpha(s) d s
\end{aligned}
$$

we get

$$
\begin{aligned}
& \left|\int_{0}^{t} \alpha(s) d s-\alpha^{*} t\right|=\left|\int_{\lfloor t\rfloor}^{t} \alpha(s) d s-\alpha^{*}(t-\lfloor t\rfloor)\right| \\
& \leq \alpha^{*}|t-\lfloor t\rfloor|+\sup _{z:|t-z| \leq 1} \int_{z}^{t} \alpha(s) d s \leq M_{0}+\alpha^{*} .
\end{aligned}
$$

From the above inequality we have

$$
-\alpha^{*}-M_{0}+\alpha^{*} t \leq \int_{0}^{t} \alpha(s) d s \leq \alpha^{*} t+\alpha^{*}+M_{0},
$$

and

$$
-\int_{0}^{t} \alpha(s) d s \leq-\alpha^{*} t+\alpha^{*}+M_{0} .
$$

We have finally the inequality

$$
\exp \left(-\int_{0}^{t} \alpha(s) d s\right) \leq e^{-\alpha^{*} t} e^{\alpha^{*}+M_{0}}=M e^{-\alpha^{*} t}
$$

By taking into account that $p(0)=(1,0,0, \ldots)^{T}$ and the form of the $1 D$-norm, where only elements of index equal or larger than 1 appear, we have that

$\|\boldsymbol{\pi}(0)-\mathbf{p}(0)\|_{1 D}=\|\boldsymbol{\pi}(0)\|_{1 D} \leq \limsup _{t \rightarrow \infty}\|\boldsymbol{\pi}(t)\|_{1 D}$.

Moreover, by (2.15) we can write

$$
\begin{aligned}
\|\pi(t)\|_{1 D}= & \|\mathbf{q}(t)\|_{1 D} \leq\|V(t, 0) \cdot \mathbf{q}(0)\|_{1 D} \\
& +\left\|\int_{0}^{t} V(t, \tau) \mathbf{f}(\tau) d \tau\right\|_{1 D}
\end{aligned}
$$

$$
\begin{aligned}
\leq & \|\mathbf{q}(0)\|_{1 D}\|V(t, 0)\|_{1 D} \\
+ & \int_{0}^{t}\|D \mathbf{f}(\tau)\|_{1}\|V(t, \tau)\|_{1 D} d \tau \quad \text { by }(2.27) \\
\leq & \|\pi(0)\|_{1 D} e^{\int_{0}^{t} \gamma(B(s))_{\ell_{1 D}} d s} \\
& +d_{0} \mathbf{a} v_{0} \int_{0}^{t} e^{\int_{\tau}^{t} \gamma(B(u))_{\ell_{1 D}} d u} d \tau \text { by }(2.28) \\
\leq & \|\pi(0)\|_{1 D} e^{-\int_{0}^{t} \alpha(s) d s}+d_{0} \mathbf{a} v_{0} \int_{0}^{t} e^{-\int_{\tau}^{t} \alpha(u) d u} d \tau \\
\leq & M e^{-\alpha^{*} t}\|\pi(0)\|_{1 D}+\operatorname{Mav} v_{0} \int_{0}^{t} e^{-\alpha^{*}(t-\tau)} d \tau,
\end{aligned}
$$

because of (2.37).

Passing to the limit as $t \rightarrow \infty$ we get

$\limsup _{t \rightarrow \infty}\|\boldsymbol{\pi}(t)\|_{1 D} \leq \frac{\mathrm{M} a v_{0}}{\alpha^{*}}$.

Finally using (2.34), (2.37), (2.38) and (2.40), we obtain (2.11).

We now consider the family of truncated BDPs $X_{N}=$ $X_{N}(t), t>0$ on the state space $E_{N}=\{0,1,2, \ldots, N\}$, where birth rates are $\lambda_{n}(t), n \in E_{N-1}$ and death rates $\mu_{n}(t)$, $n \in E_{N}$ (and with intensity matrix $\mathbf{A}_{N}$ ).

The truncated process has the vector of probabilities governed by the forward Kolmogorov differential system

$\frac{d \mathbf{p}_{N}}{d t}=A_{N}(t) \mathbf{p}_{N}$

We will identify below the finite vector with entries $\left(a_{1}, \ldots, a_{N}\right)$ and the infinite vector with the same first $N$ coordinates and the others equal to zero. The same identification will be assumed also for the rate matrix $A_{N}$.

Theorem 2. Assume there exists a sequence $\left\{d_{i}\right\}$ such that $\alpha^{*}>0$. Then for any $t \geq 0$

$$
\begin{aligned}
& \left|E\{X(t) \mid X(0)=0\}-E\left\{X_{N}(t) \mid X_{N}(0)=0\right\}\right| \\
& \quad \leq \frac{3 t M a v_{0}(L a+M b)}{W_{N} \alpha^{*}},
\end{aligned}
$$

where

$$
W_{N}=\inf _{k \geq 0} \frac{\sum_{i=0}^{k} d_{N-1+i}}{N+k} .
$$


Proof: Write the system (2.3) in the form

$\frac{d \mathbf{p}}{d t}=A_{N}(t) \mathbf{p}+\left(A(t)-A_{N}(t)\right) \mathbf{p}$.

Then

$$
\begin{aligned}
\mathbf{p}(t)= & U_{N}(t, 0) \mathbf{p}(0)+\int_{0}^{t} U_{N}(t, \tau)(A(\tau) \\
& \left.-A_{N}(\tau)\right) \mathbf{p}(\tau) d \tau .
\end{aligned}
$$

Hence

$$
\begin{aligned}
\left|E\{X(t) \mid X(0)=0\}-E\left\{X_{N}(t) \mid X_{N}(0)=0\right\}\right| \\
=\left\|\mathbf{p}(t)-\mathbf{p}_{N}(t)\right\|_{1 E} \leq \int_{0}^{t} \| U_{N}(t, \tau)(A(\tau) \\
\left.\quad-A_{N}(\tau)\right) \mathbf{p}(\tau) \|_{1 E} d \tau .
\end{aligned}
$$

We have that

$$
U_{N}=\left(\begin{array}{ccccccc}
u_{00}^{N} & \cdot & \cdot & u_{0 N}^{N} & 0 & 0 & \cdots \\
u_{10}^{N} & . & . & u_{1 N}^{N} & 0 & 0 & \cdots \\
\cdots & & & & & & \\
u_{N 0}^{N} & . & . & u_{N N}^{N} & 0 & 0 & \cdots \\
0 & . & . & 0 & 1 & 0 & \cdots \\
0 & . & . & 0 & 0 & 1 & \cdots \\
\cdots & & & & & &
\end{array}\right),
$$

and

$$
A_{N}=\left(\begin{array}{cccccccc}
-\lambda_{0} & \mu_{1} & 0 & \cdots & 0 & 0 & 0 & \cdots \\
\lambda_{0} & -\left(\lambda_{1}+\mu_{1}\right) & \mu_{2} & \cdots & 0 & 0 & 0 & \cdots \\
\cdots & & & & & & & \\
\cdots & & & & & & & \\
0 & 0 & 0 & \cdots & \lambda_{N-1} & -\mu_{N} & 0 & \cdots \\
0 & 0 & \cdots & & 0 & 0 & \ddots & \\
\vdots & \vdots & & & & & & \ddots
\end{array}\right) .
$$

Then

$$
\begin{aligned}
\left(A-A_{N}\right) \mathbf{p}= & \left(0, \ldots, 0,-\lambda_{N} p_{N}+\mu_{N+1} p_{N+1}, \lambda_{N} p_{N}\right. \\
& \left.-\left(\lambda_{N+1}+\mu_{N+1}\right) p_{N+1}+\mu_{N+2} p_{N+2}, \ldots\right)^{T},
\end{aligned}
$$

and

$$
\begin{aligned}
& U_{N}\left(A-A_{N}\right) \mathbf{p} \\
& =\left(\begin{array}{c}
u_{0 N}^{N}\left(-\lambda_{N} p_{N}+\mu_{N+1} p_{N+1}\right) \\
u_{1 N}^{N}\left(-\lambda_{N} p_{N}+\mu_{N+1} p_{N+1}\right) \\
\vdots \\
u_{N N}^{N}\left(-\lambda_{N} p_{N}+\mu_{N+1} p_{N+1}\right) \\
\lambda_{N} p_{N}-\left(\lambda_{N+1}+\mu_{N+1}\right) p_{N+1}+\mu_{N+2} p_{N+2} \\
\vdots
\end{array}\right)
\end{aligned}
$$

Inequalities $u_{i j}^{N}(t, \tau) \geq 0$ for all $i, j, t, \tau$ and equalities $\sum_{i} u_{i j}^{N}(t, \tau)=1$ for all $j, t, \tau$ imply the bound

$$
\left\|U_{N}\left(A-A_{N}\right) \mathbf{p}\right\|_{1 E}=\left|-\lambda_{N} p_{N}+\mu_{N+1} p_{N+1}\right| \sum_{n \leq N} n u_{n N}^{N}
$$

$$
+\sum_{k \geq N}(k+1)\left|\lambda_{k} p_{k}-\left(\lambda_{k+1}+\mu_{k+1}\right) p_{k+1}+\mu_{k+2} p_{k+2}\right|
$$$$
\leq\left(L \mathrm{a} p_{N}+M \mathrm{~b} p_{N+1}\right)
$$$$
\times\left(\sum_{k=0}^{N-1}(k-(k+1)) \sum_{i=0}^{k} u_{i N}^{N}+N \sum_{i=0}^{N} u_{i N}^{N}\right)
$$$$
+\sum_{k \geq N}(k+1)\left(L a\left(p_{k}+p_{k+1}\right)\right.
$$$$
\left.+M \mathrm{~b}\left(p_{k+1}+p_{k+2}\right)\right)
$$$$
\leq \mathrm{La}\left(N p_{N}+(N+1)\left(p_{N}+p_{N+1}\right)+(N+2)\left(p_{N+1}\right.\right.
$$$$
\left.\left.+p_{N+2}\right)+\cdots\right)+M \mathrm{~b}\left(N p_{N+1}+(N+1)\left(p_{N+1}\right.\right.
$$$$
\left.\left.+p_{N+2}\right)+(N+2)\left(p_{N+2}+p_{N+3}\right)+\cdots\right) \leq
$$$$
\leq(L a+M b) \sum_{k \geq N}(2 k+1) p_{k} \leq 3(L a+M b) \sum_{k \geq N} k p_{k} \text {. }
$$

By (2.39) (with $\pi(t)$ replaced by $\mathbf{z}(t)$ ) and assuming that $\mathbf{z}(0)=\mathbf{0}$ we get

$\|\mathbf{z}(t)\|_{1 D} \leq \frac{\mathrm{M} a v_{0}}{\alpha^{*}}$

On the other hand,

$$
\begin{aligned}
& d_{N-1}\left(p_{N}+p_{N+1}+\ldots\right)+d_{N}\left(p_{N+1}+p_{N+2}+\ldots\right)+\ldots \\
& \quad=p_{N} d_{N-1}+p_{N+1}\left(d_{N-1}+d_{N}\right)+p_{N+2}\left(d_{N-1}\right. \\
& \left.\quad+d_{N}+d_{N+1}\right)+\ldots
\end{aligned}
$$




$$
\begin{aligned}
= & \frac{d_{N-1}}{N} N p_{N}+\frac{d_{N-1}+d_{N}}{N+1}(N+1) p_{N+1} \\
& +\cdots \geq \mathbf{W}_{N} \sum_{k \geq N} k p_{k} .
\end{aligned}
$$

Then

$$
\begin{aligned}
\sum_{k \geq N} k p_{k} & \leq W_{N}^{-1} \sum_{k \geq N} d_{k-1}\left(\sum_{i \geq k} p_{i}\right) \leq W_{N}^{-1}\|\mathbf{z}\|_{1 D} \\
& \leq \frac{\mathrm{M} a v_{0}}{\mathrm{~W}_{N} \alpha^{*}} .
\end{aligned}
$$

Finally from (2.46), (2.51), (2.52) and (2.54) we obtain (2.42).

As a consequence of Theorem 1 and Theorem 2 we obtain the following statement.

Corollary 1. Let $\left\{d_{i}\right\}$ be a sequence such that $\alpha^{*}>0$. Then for any $t \geq 0$

$$
\begin{aligned}
\mid \phi(t)- & E\left\{X_{N}(t) \mid X(0)=0 .\right\} \mid \leq \frac{\mathrm{M}^{2} a v_{0}}{W \alpha^{*}} e^{-\alpha^{*} t} \\
+ & \frac{3 t \mathrm{M} a v_{0}(L a+\mathrm{M} b)}{\mathrm{W}_{N} \alpha^{*}} .
\end{aligned}
$$

Remark 1. Here the first expression of the right-hand side of (2.55) tends to zero as $t \rightarrow \infty$, and the second expression tends to zero as $N \rightarrow \infty$ (for any fixed $t$, provided that $W_{N} \rightarrow \infty$ as $N \rightarrow \infty$ ). Hence, Theorem 2 gives us a tool for calculating the limiting mean, as shown in the examples below.

Let now introduce the following important characteristic of the nonhomogeneous BDP.

Definition 2. The double mean of the BDP $X=X(t), t \geq 0$ is defined by

$E=\lim _{t \rightarrow \infty} \frac{1}{t} \int_{0}^{t} E\{X(u) \mid X(0)=k\} d u$,

provided that the limit exists and does not depend on $k$.

Theorem 3. Let $\left\{d_{i}\right\}$ be a sequence such that $\alpha^{*}>0$ and assume also that $\inf _{k \geq 1} \frac{d_{k-1}}{k}=\omega>0$. Then the double mean of the BDP X exists and the following inequalities hold:

$\left|\mathrm{E}-\int_{t}^{t+1} E\{X(u) \mid X(0)=0\} d u\right| \leq \frac{\mathrm{M}^{2} a v_{0}}{\mathrm{~W} \alpha^{*}} e^{-\alpha^{*} t}$, and

$$
\begin{aligned}
\mid \mathrm{E}- & \int_{t}^{t+1} E\left\{X_{N}(u) \mid X_{N}(0)=0\right\} d u \mid \leq \frac{\mathrm{M}^{2} a v_{0}}{\mathrm{~W} \alpha^{*}} e^{-\alpha^{*} t} \\
& +\frac{3(t+1) \mathrm{M} a v_{0}(L a+\mathrm{M} b)}{\mathrm{W}_{N} \alpha^{*}}
\end{aligned}
$$

Proof: The existence of the double mean follows from Theorem 1 which gives the bound

$\left|\frac{1}{t} \int_{0}^{t}(E\{X(u) \mid X(0)=0\}-\phi(u)) d u\right| \leq \frac{\mathrm{M}^{2} a v_{0}}{t \mathrm{~W} \alpha^{* 2}}$.

Convergence to zero of (2.59) and 1-periodicity of $\phi$, imply that

$\mathrm{E}=\lim _{t \rightarrow \infty} \frac{1}{t} \int_{0}^{t} \phi(u) d u=\int_{0}^{1} \phi(u) d u$.

By applying again Theorem 1, we obtain

$$
\begin{gathered}
\left|\mathrm{E}-\int_{t}^{t+1} E\{X(u) \mid X(0)=0\} d u\right|=\mid \int_{t}^{t+1}(\phi(u) \\
-E\{X(u) \mid X(0)=0\}) d u \mid \leq \frac{M^{2} a v_{0}}{W \alpha^{*}} e^{-\alpha^{*} t} .
\end{gathered}
$$

Corollary 1 gives (2.58):

$$
\begin{aligned}
\mid \mathrm{E}- & \int_{t}^{t+1} E\left\{X_{N}(u) \mid X_{N}(0)=0\right\} d u \mid \\
& =\left|\int_{t}^{t+1}\left(\phi(u)-E\left\{X_{N}(u) \mid X_{N}(0)=0\right\}\right) d u\right| \\
& \leq \frac{\mathrm{M}^{2} a v_{0}}{\mathrm{~W} \alpha^{*}} e^{-\alpha^{*} t}+\frac{3(t+1) \mathrm{M} a v_{0}(L a+M \mathrm{~b})}{\mathrm{W}_{N} \alpha^{*}} .
\end{aligned}
$$

Remark 2. If the basic functions $a(t), b(t)$ are $T$-periodic with $T \neq 1$, then all the bounds of the theorems above must be changed according to

$$
\begin{gathered}
\alpha^{*}=\frac{1}{T} \int_{0}^{T} \alpha(t) d t, \quad M_{0}=\sup _{|t-s| \leq T} \int_{s}^{t} \alpha(u) d u, \\
\mathrm{M}=e^{M_{0}+\alpha^{*} T},
\end{gathered}
$$

in place of (2.11). 


\section{Some examples}

We consider the simplest nonhomogeneous queueing systems, with periodic rates.

We show how the limiting mean length of the queue (a periodic function of time $t$ ) is obtained. The same construction for the double mean is also presented.

In our examples we choose some values of the period $T$ corresponding to different situations: the case $T=1$ is related to the standard situation examined above, the case of a short period $T=0.01$ corresponds to rapidly varying processes and the case of a long period $(T=100)$ approximates the situation where the intensity of arrivals of customers and the duration of services significantly differ.

It is interesting to remark that the behaviour of the limiting averages in the models $M(t) / M(t) / 1$ and $M(t) /$ $M(t) / 2$ is simpler for small and intermediate values of the period.

In these cases only negligible oscillations of the mean values are perceived during the period.

For large values of $T$ in the arrival and service rates the oscillations in the limiting mean values are instead extremely significant.

The choice of the weights $d_{i}$ considerably influences the different estimates of the speed of convergence.

The optimal choice of the sequence $\left\{d_{i}\right\}$ in the general case is a difficult problem which will be examined in a separate paper.

The program used for the calculation of the limiting averages and the limiting mean was written in Delphi 6, while the calculations have been carried out with a processor Celeron 2400 .

Example 1. (Queue-length process for the $M(t) / M(t) / 1$ queue). We present different examples of periodic func- tions $a(t)$ and $b(t)$. (i) $a(t)=1+\sin 2 \pi t$ and $b(t)=$ $4+2 \cos 2 \pi t$ (period $T=1)$. We have $v_{n}=\eta_{n}=1$, $L=M=1$ and $a=2, \quad b=6$. Set $d_{k}=2^{k}, \quad k \geq 0$. Then $\alpha(t)=1-\sin 2 \pi t+\cos 2 \pi t, \alpha^{*}=\int_{0}^{1} \alpha(t) d t=1$, $M_{0}=\sup _{|t-s| \leq 1} \int_{s}^{t} \alpha(u) d u=1+\frac{1}{2 \pi}$ and $M=e^{M_{0}+\alpha^{*}}=$ $e^{2+\frac{1}{2 \pi}}<10$. Moreover, $W=\inf _{k} \frac{\sum_{i=0}^{k-1} d_{i}}{k}=1$ and $W_{N}=$ $\inf _{k \geq 0} \frac{\sum_{i=0}^{k} 0^{N-1+i}}{N+k}=\frac{2^{N-1}}{N}$.

Hence by Theorem 1, Theorem 2 and Corollary 1, we have that

$$
\begin{aligned}
& |E\{X(t) \mid X(0)=0\}-\phi(t)| \leq 200 e^{-t} \\
& \left|E\{X(t) \mid X(0)=0\}-E\left\{X_{N}(t) \mid X_{N}(0)=0\right\}\right| \leq \frac{480 N t}{2^{N-1}}
\end{aligned}
$$

and

$\left|\phi(t)-E\left\{X_{N}(t) \mid X_{N}(0)=0\right\}\right| \leq 200 e^{-t}+\frac{480 N t}{2^{N-1}}$.

Theorem 3 implies that $\left|E-\int_{t}^{t+1} E\{X(u) \mid X(0)=0\} d u\right| \leq$ $200 e^{-t}$, and $\left|E-\int_{t}^{t+1} E\left\{X_{N}(u) \mid X_{N}(0)=0\right\} d u\right| \leq 200$ $e^{-t}+\frac{480 N(t+1)}{2^{N-1}}$.

For $t=24, N=50$, the above inequality writes $\mid E-$ $0.38305460 \mid \leq 10^{-8}$.

In figure 1 the mean $E\left\{X_{N}(t) \mid X_{N}(0)=0\right\}$ when $t$ ranges from 24 to 25 is drawn.

(ii) $a(t)=1+\sin 200 \pi t, b(t)=4+2 \cos 200 \pi t$ (period $T=0.01) . v_{n}=\eta_{n}=1=L=M, a=2$ and $b=6$. For $d_{k}=2^{k}, k \geq 0, \alpha(t)=1-\sin 200 \pi t+2 \cos 200 \pi t ; \alpha^{*}=$ $100 \int_{0}^{0.01} \alpha(t) d t=1, M_{0}=\sup _{|t-s| \leq 0.01} \int_{s}^{t} \alpha(u) d u=0.01$ $+\frac{1}{200 \pi}$ and $M=e^{M_{0}+T \alpha^{*}}=e^{0.02+\frac{1}{200 \pi}}<1.1 . \quad W=\inf _{k}$ $\frac{\sum_{i=0}^{k-1} d_{i}}{k}=1$ and $W_{N}=\inf _{k \geq 0} \frac{\sum_{i=0}^{k} 2^{N-1+i}}{N+k}=\frac{2^{N-1}}{N}$.
Fig. $1 E\left\{X_{N}(t) \mid X_{N}(0)=0\right\}$, for $t \in[24,25]$.

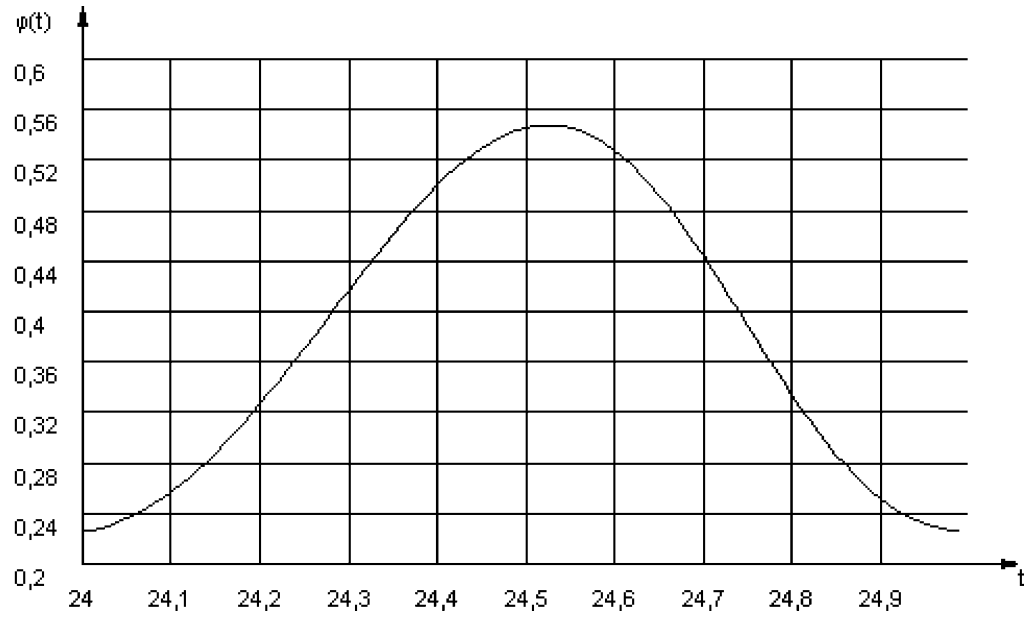


Fig. $2 E\left\{X_{N}(t) \mid X_{N}(0)=0\right\}$, for $t \in[20,20.01]$.

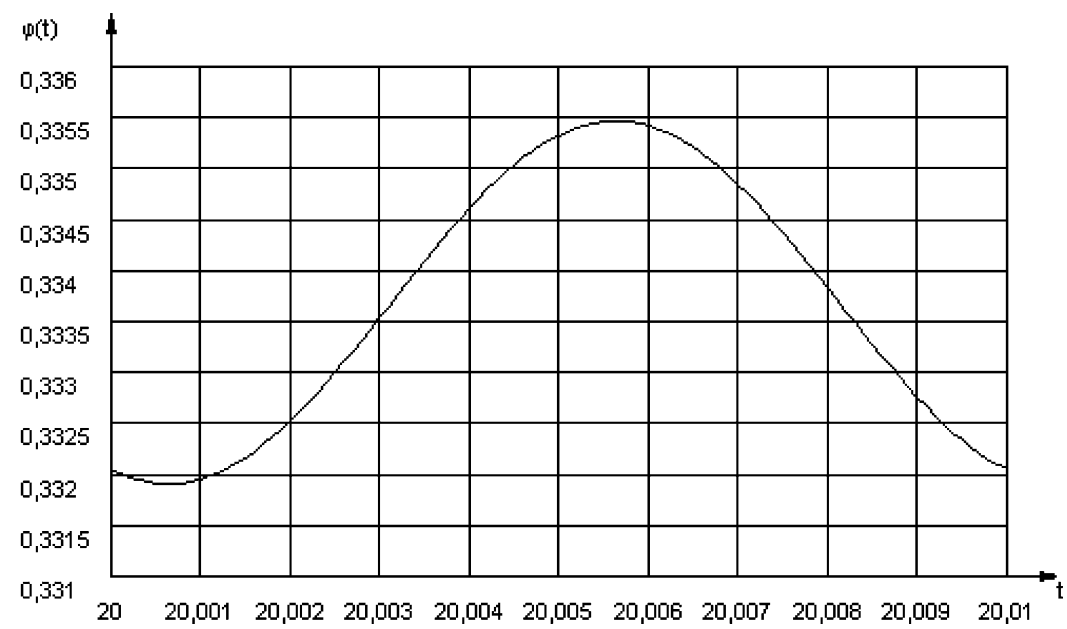

Hence

$$
\begin{aligned}
& |E\{X(t) \mid X(0)=0\}-\phi(t)| \leq 3 e^{-t} \quad \mid E\{X(t) \mid X(0)=0\} \\
& -E\left\{X_{N}(t) \mid X_{N}(0)=0\right\} \mid \leq \frac{53 N t}{2^{N-1}} \\
& \left|\mathrm{E}-100 \int_{t}^{t+0.01} E\left\{X_{N}(u) \mid X_{N}(0)=0\right\} d u\right| \leq 3 e^{-t} \\
& \quad+\frac{53 N(t+1)}{2^{N-1}} .
\end{aligned}
$$

Then, for $t=20, N=45$, we have that

$$
|E-0.33354528| \leq 10^{-8}
$$

In figure 2 the mean $E\left\{X_{N}(t) \mid X_{N}(0)=0\right\}$, for $t$ belonging to the interval $[20,20.01]$ is pictured. (iii) $\quad a(t)=1+\sin 0.02 \pi t, \quad b(t)=4+2 \cos 0.02 \pi t$. With the same choice for $\left\{d_{k}\right\}$ we get the estimates $|E\{X(t) \mid X(0)=0\}-\phi(t)| \leq 2 e^{432-t}, \quad \mid E\{X(t) \mid X(0)=0\}$ $-E\left\{X_{N}(t) \mid X_{N}(0)=0\right\} \mid \leq \frac{48 \cdot e^{216} N t}{2^{N-1}}$, and $\mid E-0.01 \int_{t}^{t+100}$ $E\left\{X_{N}(u) \mid X_{N}(0)=0\right\} d u \mid \leq 2 e^{432-t}+\frac{48 \cdot e^{216} N(t+1)}{2^{N-1}}$.

For $t=455, \quad N=365$, the above yields

$|E-0.60725144| \leq 10^{-8}$

Figure 3 represents the mean $E\left\{X_{N}(t) \mid X_{N}(0)=0\right\}$ for $t \in[455,555]$.

Example 2. (Queue-length process for the $M(t) / M(t) / 2$ queue). We consider now the same periodic functions of Example 1.

(i) $a(t)=1+\sin 2 \pi t, b(t)=4+2 \cos 2 \pi t \quad$ (1-periodic functions). In this case we have $v_{n}=\eta_{1}=1(n \geq 1), \eta_{n}=$
Fig. $3 E\left\{X_{N}(t) \mid X_{N}(0)=0\right\}$, for $t \in[455,555]$.

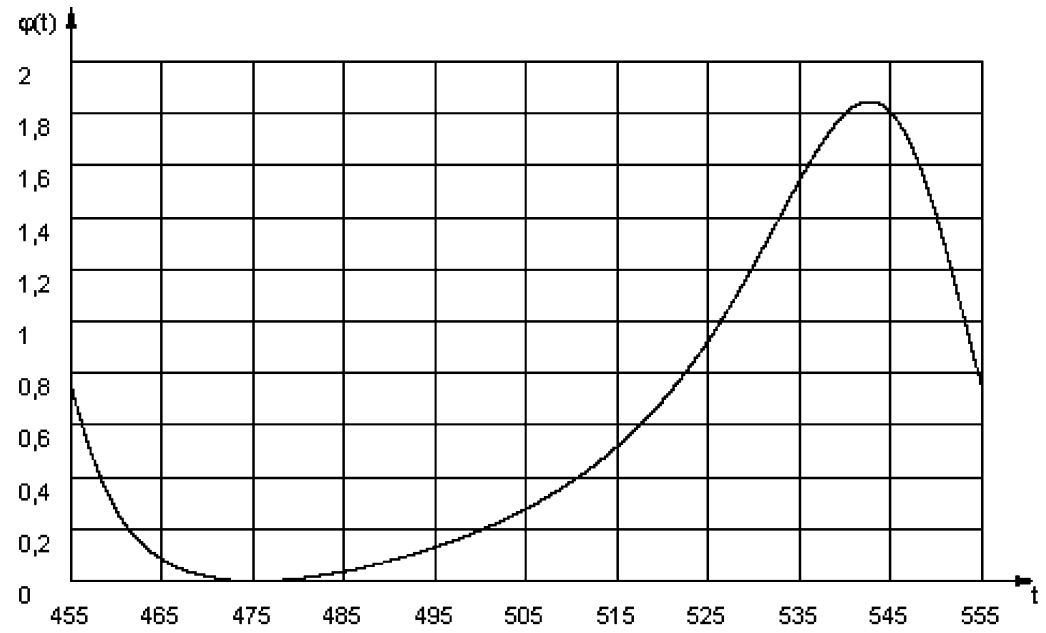


Fig. $4 E\left\{X_{N}(t) \mid X_{N}(0)=0\right\}$, for $t \in[11,12]$.

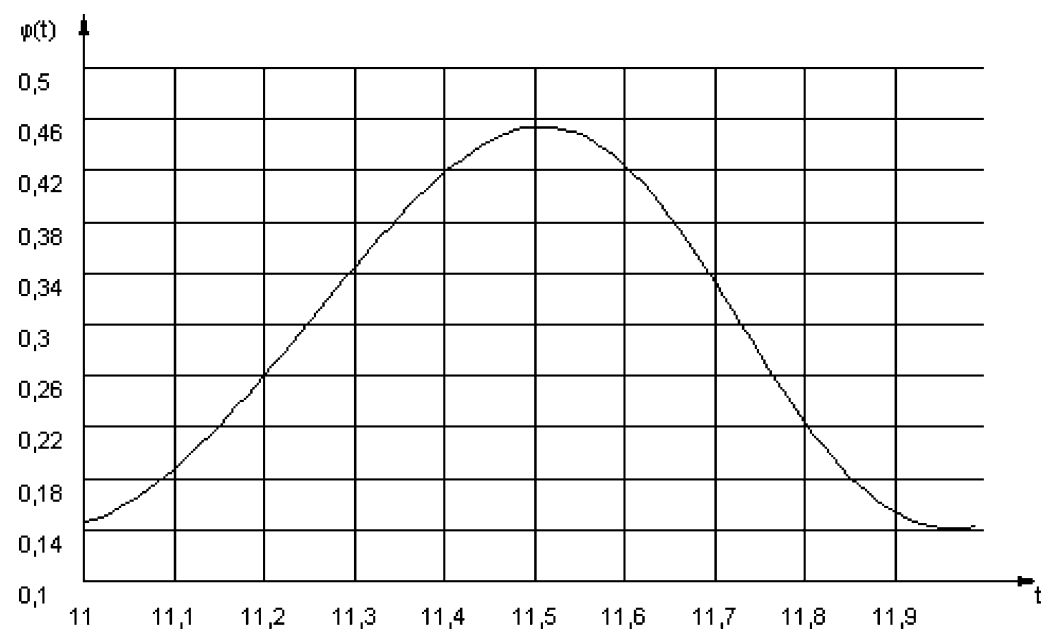

$$
\begin{aligned}
\mid \mathrm{E}- & 100 \int_{t}^{t+0.01} E\left\{X_{N}(u) \mid X_{N}(0)=0\right\} d u \mid \leq 6 e^{-3 t} \\
& +\frac{72 N(t+1)}{2^{N-1}} .
\end{aligned}
$$

Set $t=7, N=44$. Then the last inequality becomes

$$
\begin{aligned}
& |E\{X(t) \mid X(0)=0\}-\phi(t)| \leq 2 \cdot 10^{5} e^{-3 t} \\
& \left|E\{X(t) \mid X(0)=0\}-E\left\{X_{N}(t) \mid X_{N}(0)=0\right\}\right| \leq \frac{3 \cdot 10^{4} N t}{4 \cdot 2^{N-2}}, \\
& \left|\phi(t)-E\left\{X_{N}(t) \mid X_{N}(0)=0\right\}\right| \leq 2 \cdot 10^{5} e^{-3 t}+\frac{3 \cdot 10^{4} N t}{4 \cdot 2^{N-2}}, \\
& \quad \text { and, by Theorem } 3 \\
& \left|\mathrm{E}-\int_{t}^{t+1} E\{X(u) \mid X(0)=0\} d u\right| \leq 2 \cdot 10^{5} e^{-3 t}, \\
& \left|\mathrm{E}-\int_{t}^{t+1} E\left\{X_{N}(u) \mid X_{N}(0)=0\right\} d u\right| \leq 2 \cdot 10^{5} e^{-3 t} \\
& \quad+\frac{3 \cdot 10^{4} N(t+1)}{4 \cdot 2^{N-2}},
\end{aligned}
$$

Put $t=11, N=54$. The last bound then becomes $|E-0.29456925| \leq 10^{-8}$. In figure 4 the function $E\left\{X_{N}(t) \mid X_{N}(0)=0\right\}$ for $t$ inside [11, 12] is drawn.

(ii) $a(t)=1+\sin 200 \pi t, b(t)=4+2 \cos 200 \pi t$. Then $v_{n}=\eta_{1}=1(n \geq 1), \eta_{n}=2(n \geq 2), L=1, M=2, a=$ $2, \quad b=6$. For $d_{k}=2^{k}, k \geq 1$, we obtain the following bounds

$$
\begin{aligned}
& |E\{X(t) \mid X(0)=0\}-\phi(t)| \leq 6 e^{-3 t} \\
& \left|E\{X(t) \mid X(0)=0\}-E\left\{X_{N}(t) \mid X_{N}(0)=0\right\}\right| \leq \frac{72 N t}{2^{N-1}}
\end{aligned}
$$

$|E-0.25434930| \leq 10^{-8}$

Figure 5 shows the mean $E\left\{X_{N}(t) \mid X_{N}(0)=0\right\}$ in the interval [7, 7.01].

(iii) $a(t)=1+\sin 0.02 \pi t, b(t)=4+2 \cos 0.02 \pi t$ (period $T=100$ ). In this case we obtain (for the same sequence $d_{k}=2^{k}$ ) the bounds:

$$
\begin{aligned}
& |E\{X(t) \mid X(0)=0\}-\phi(t)| \leq e^{1232-3 t}, \\
& \left|E\{X(t) \mid X(0)=0\}-E\left\{X_{N}(t) \mid X_{N}(0)=0\right\}\right| \\
& \quad \leq \frac{40 N t e^{616}}{2^{N-1}}, \\
& \left|\mathrm{E}-0.01 \int_{t}^{t+100} E\left\{X_{N}(u) \mid X_{N}(0)=0\right\} d u\right| \leq e^{1232-3 t} \\
& \quad+\frac{40 N(t+1) e^{616}}{2^{N-1}},
\end{aligned}
$$

which, for $t=418$ and $N=945$ yields

$|E-0.31032003| \leq 10^{-8}$. 
Fig. $5 E\left\{X_{N}(t) \mid X_{N}(0)=0\right\}$, for $t \in[7,7.01]$.

Fig. $6 E\left\{X_{N}(t) \mid X_{N}(0)=0\right\}$, for $t \in[418,518]$.
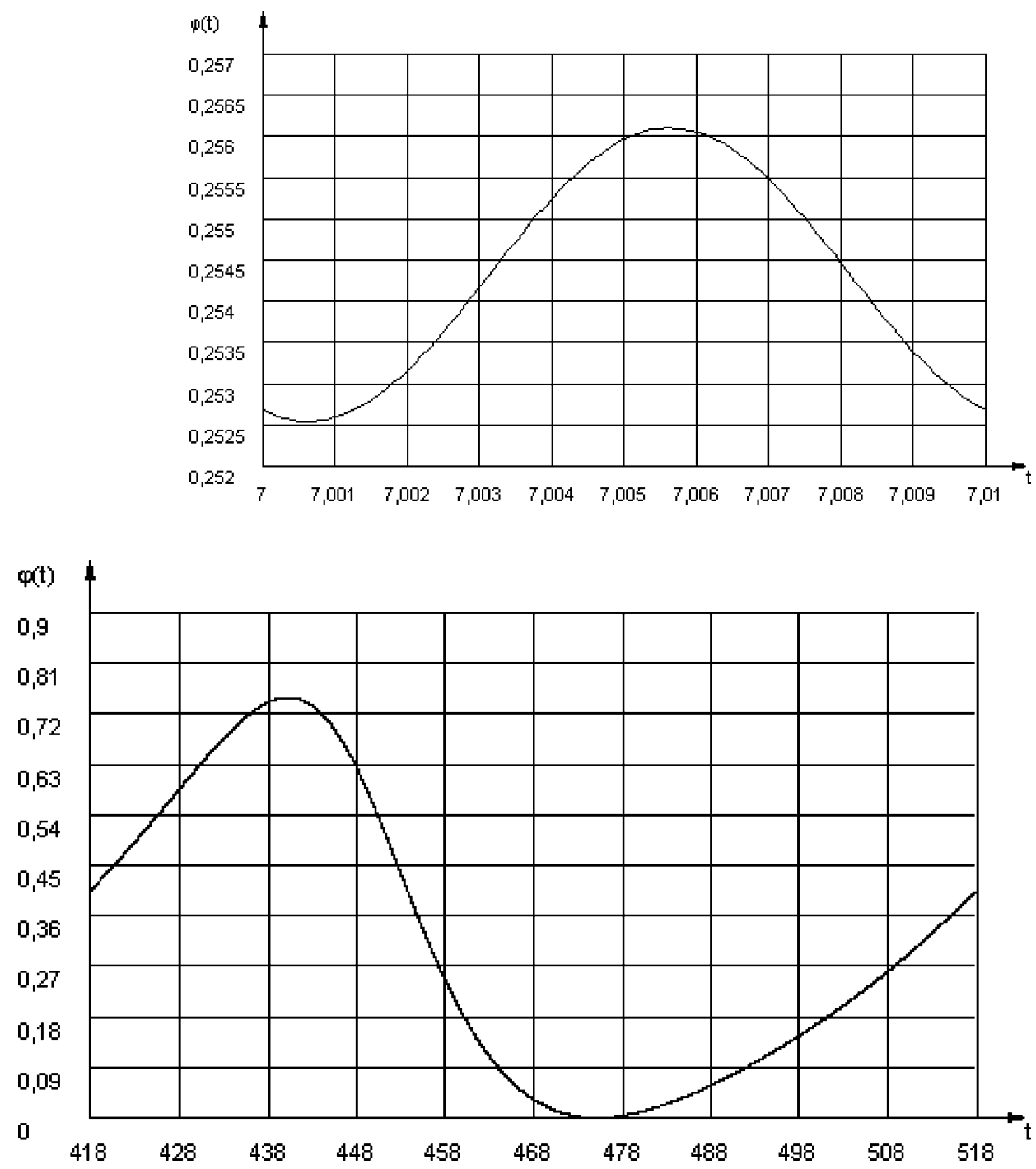

The function $E\left\{X_{N}(t) \mid X_{N}(0)=0\right\}$, for $t \in[418,518]$, is represented in figure 6 .

Acknowledgments The paper was begun in summer 2003 during the visit of Prof. A.I. Zeifman to the Dipartimento di Statistica, Probabilità e Statistiche Applicate of University of Rome "La Sapienza", to which he wants to express his thanks for the financial support received. We want to thank the Referee and the Associate Editor for very useful remarks.

\section{References}

1. Y. Daletsky and M.G. Krein, Stability of solutions of differential equations in Banach spaces, Ann. Math. Soc. Transl. 43 (1974) 386. American Mathematical Society, Providence, R.I.

2. A. Di Crescenzo and A.G. Nobile, Diffusion approximation to a queueing system with time dependent arrival and ser- vice rates, Queueing Systems Theory Appl. 19 (1995) 4162.

3. B.V. Gnedenko and I.P. Makarov, Properties of a problem with losses in the case of periodic intensities, Diff. Equations 7 (1971) 1696-1698 (in Russian).

4. B. Granovsky and A. Zeifman, The $N$-limit of spectral gap of a class of birth-death Markov chains, Appl. Stoch. Models in Business and Industry 16 (2000) 235-248.

5. B. Granovsky and A. Zeifman, Nonstationary queues: Estimation of the rate of convergence, Queueing Systems 46 (2004) 363388.

6. A. Mandelbaum and W. Massey, Strong approximations for timedependent queues, Math. Oper. Res. 20 (1995) 33-64.

7. B. Margolius, A sample path analysis of the $M_{t} / M_{t} / c$ queue, Queueing Systems Theory Appl. 31(1-2) (1999) 59-93.

8. W.A. Massey and W. Whitt, On analysis of the modified offeredload approximation for the nonstationary Erlang loss model. Ann. Appl. Probab. 4 (1994) 1145-1160.

9. F. Simonot, Sur l'approximation de la distribution stationnaire d'une chaine de Markov stochastiquement monotone, Stoch. Proc. Appl. 56(1) (1995) 137-149. 
10. A.I. Zeifman, Stability for continuous-time nonhomogeneous Markov chains, Lect. Notes Mathem. 1155 (1985) 401-414.

11. A.I. Zeifman, Truncation error in a birth and death system, U.S.S.R. Comput. Math. and Math. Phys. 28(6) (1988) 210 211.
12. A.I. Zeifman, On the estimation of probabilities for birth and death processes, J. Appl. Probab. 32 (1995) 623-634.

13. A.I. Zeifman, Upper and lower bounds on the rate of convergence for nonhomogeneous birth and death processes, Stoch. Proc. Appl. 59 (1995) 157-173. 